\title{
Ansiedad matemática en estudiantes universitarios de Costa Rica y su relación con el rendimientos académico y variables sociodemográficas
}

\author{
Mathematics Anxiety in College Students in Costa Rica and their \\ Relatonship with Academic Achievement and Socio-Demographic \\ Variables
}

\section{Islande C. Delgado Monge ${ }^{1 *}$, Johan Espinoza González ${ }^{2}$ \& Jennifer Fonseca Castro $^{2}$}

${ }^{1}$ Universidad Estatal a Distancia de Costa Rica, San José, Costa Rica.

${ }^{2}$ Universidad Nacional de Costa Rica, Heredia, Costa Rica.

\author{
Recibido 11-01-17 \\ Aprobado 19-03-17
}

\section{Correspondencia}

Email: idelgado@uned.ac.cr
Citar como:

Delgado, I., Espinoza, J. \& Fonseca, J. (2017). Ansiedadmatemática en estudiantes universitarios de Costa Rica y su relación con el rendimientos. académico y variables sociodemográficas. Propósitos y Representaciones, 5(1), 275 324 doi: http://dx.doi.org/10.20511/pyr2017. v5n1.148

(C) Universidad San Ignacio de Loyola, Vicerrectorado de Investigación y Desarrollo, 2017. (cc) BY-NC-ND Este artículo se distribuye bajo licencia CC BY-NC-ND 4.0 Internacional (http://creativecommons.org/licenses/by-nc-nd/4.0/). 


\section{Resumen}

El estudio buscó determinar la relación entre ansiedad matemática y las variables género, rendimiento académico, cantidad de veces que ha llevado el curso y tipo de colegio, en estudiantes del curso MAT-001 Matemática General de la Universidad Nacional de Costa Rica. Para ello se escogió una muestra intencional de 472 alumnos de dicho curso y se les aplicó una adecuación del instrumento de Hopko, Mahadevan, Bare y Hunt (2003). Los datos recolectados fueron analizados con el programa estadístico SPSS aplicando las pruebas de hipótesis correspondientes. De acuerdo con los resultados, se encontró que los estudiantes presentaron un nivel medio de ansiedad Matemática y que las mujeres mostraron una ansiedad matemática significativamente mayor que los hombres; mientras que los estudiantes egresados de colegios privados obtuvieron una ansiedad matemática significativamente menor que los provenientes de colegios públicos o de la modalidad de "bachillerato por madurez". De igual forma resultó que entre mayor es la ansiedad matemática del estudiante su rendimiento académico será significativamente menor. Por último, no se encontraron diferencias estadísticamente significativas entre la ansiedad matemática y la cantidad de veces que el estudiante ha llevado el curso.

Palabras clave: ansiedad matemática; estudiantes universitarios; rendimiento académico; variables sociodemográficas.-

\section{Summary}

The study tried to determine the relationship between mathematics anxiety and the variables of gender, academic achievement, number of times students have taken the course and type of school in students taking the course MAT001 General Mathematics of the National University of Costa Rica. To that end, a purposive sample of 472 students of such course was collected and the instrument of Hopko, Mahadevan, Bare and Hunt was adjusted to it (2003). Data collected were analyzed with the statistical program SPSS applying the corresponding hypothesis tests. According with the results, students showed a 
medium level of math anxiety and women showed a math anxiety significantly higher than men, while student graduated from private schools showed a significantly lower level of math anxiety than those from public schools or from the modality "General Equivalency Diploma". Besides, the higher the math anxiety of the student is, the lower their academic achievement will be. Finally, there were no statistically significant differences between math anxiety and the number of times students have taken the course.

Keywords: Mathematics anxiety, college students, academic achievement, socio-demographic variables 


\section{Introducción}

El aprendizaje de las matemáticas son cada vez más importantes, así lo indica el Consejo Nacional de Profesores de Matemática (NCTM, 2003) al mencionar que "en este mundo cambiante aquellos que comprendan y puedan hacer y usar matemáticas tendrán cada vez más oportunidades y opciones para determinar su futuro" (p.5).

Sin embargo, constantemente se escucha decir a los estudiantes frases como: "no me gusta la Matemática", "la Matemática es muy difícil", "la Matemática me hace sentir nervioso y preocupado", "quiero una carrera que no incluya cursos de Matemática", entre otras. Estas referencias podrían ser síntomas de lo que algunos expertos han denominado ansiedad matemática. Al respecto, Marshall (2000), indica que la ansiedad matemática "es una importante, pero poco entendida realidad para los estudiantes y un aspecto dirigido de manera descuidada por parte de algunos profesores" (p. 108).

Pérez-Tyteca y Castro (2011) también hacen referencia a esta conducta y la describen como un estado afectivo caracterizado por la ausencia de confort, la cual se manifiesta mediante una serie de "síntomas", tales como: tensión, nervios, preocupación, inquietud, irritabilidad, impaciencia, confusión, miedo y bloqueo mental.

Este es un tema que ha sido de interés por la comunidad de investigadores desde hace más de 40 años, y es tal que aún continúa siendo un tema de gran relevancia. Evidencia de ello es su incorporación en el estudio PISA 2003 implementado en 40 países, cuyos resultados reflejan que una gran cantidad de los estudiantes de 15 años que participaron en el estudio presentaron ansiedad matemática (Pérez-Tyteca, 2012).

De igual forma, se ha reconocido y resaltado el dominio afectivo como un aspecto de gran importancia en el proceso de enseñanza y aprendizaje de la Matemática, de manera que algunos autores indican que es necesario disminuir el temor que sienten los estudiantes hacia la matemática, previo 
a enseñársela (Guerrero, Blanco \& Castro, 2001; Pérez-Tyteca \& Castro, 2011; Pérez-Tyteca, Castro, Rico \& Castro, 2011; Quiles, 1993).

Además, Manay (2009, como se citó en Lamas, 2015) indica que existe una relación significativa entre la ansiedad y rendimiento académico. En este sentido, algunos autores afirman que la ansiedad matemática presenta efectos en los estudiantes en cuanto a su rendimiento académico (Gil, Blanco \& Guerrero, 2005; Mato \& Muñoz, 2010; Pérez-Tyteca \& Castro, 2011; Manay, 2009; como se citó en Lamas, 2015), ya que una alta ansiedad matemática está ligada a un bajo rendimiento académico y viceversa (Aiken, 1970; Reyes, 1984).

De la misma manera, se ha observado que la ansiedad matemática puede influir en la elección de la carrera universitaria por parte del estudiante, pues un bajo rendimiento en esta materia puede limitarlos a elegir carreras en las cuales el plan de estudios contenga la menor cantidad de cursos de matemática (Pérez-Tyteca \& Castro, 2011).

Esta conducta también repercute en el comportamiento del alumno cuando trabaja con contenidos de matemática ya que una ansiedad excesiva produce una disminición en las energías y atención de los estudiantes hacia actividades cognitivas (Alegre, 2013). Además, los estudiantes que presentan un alto nivel prefieren realizar las operaciones lo más rápido posible, dejando de lado el cuidado que se debe tener al desarrollarlas (Hackett, 1985). De acuerdo con Ashcraft y Krause (2007), realizan estas acciones con el fin de disminuir el tiempo que la sufren.

Así, al tomar en cuenta que en el nivel universitario es donde mayoritariamente se manifiesta en los estudiantes la problemática de la ansiedad matemática (Gavira, 2008) y que esta presenta algunos efectos negativos en el rendimiento académico y en el comportamiento general del estudiante cuando se enfrenta a tareas matemáticas, es que se considera relevante realizar un estudio con un grupo de estudiantes universitarios para valorar el nivel de ansiedad matemática y su relación con algunas variables. 
Para ello se eligió un curso introductorio de matemática dictado en la Universidad Nacional de Costa Rica llamado MAT-001 Matemática General, el cual sirve de base para las asignaturas de Cálculo Diferencial e Integral y Cálculo I, y es el único curso de matemática que estudiantes de 13 titulaciones cursan para su formación profesional. Es importante mencionar que dicho curso se caracteriza por tener un porcentaje de aprobación de un 35\%.

Por tanto, se plantea la siguiente pregunta de investigación ¿Cuál es el nivel de ansiedad matemática que muestran los estudiantes del curso MAT001 Matemática General de la Universidad Nacional de Costa Rica y su relación con las variables: (a) sexo; (b) rendimiento académico; (c) tipo de colegio del cual es egresado el estudiante; (d) horario del curso; y (e) cantidad de veces que el alumno ha llevado el curso.

\section{Definiciones de ansiedad matemática.}

Al investigar sobre la definición de ansiedad matemática se puede constatar que existe una diversidad de conceptos; sin embargo, todos estos están relacionados y siguen una misma línea.

Fennema y Sherman (1976), añaden que la ansiedad matemática "consiste en una serie de sentimientos de ansiedad, terror, nerviosismo y síntomas físicos que surgen al hacer matemáticas" (p.4).

Por su parte, Richardson y Suinn (1972), la definen como el "sentimiento de tensión y ansiedad que interfieren en la manipulación de números y en la resolución de problemas matemáticos en una amplia variedad de situaciones tanto cotidianas como académicas" (p.551).

Así mismo, Tobías y Weissbroad (1980), manifiestan que "la ansiedad matemática describe el pánico, indefensión, parálisis y desorganización mental que surgen cuando a un sujeto se le exige resolver un problema matemático" (p.65).

Más específicamente, Perry (2004) define diferentes tipos de ansiedad matemática en alumnos universitarios: (a) ansiedad matemática moderada y 
variante; (b) ansiedad matemática en el estudiante desde tiempo atrás y que inició como consecuencia de la actuación de algún profesor; y (c) la causada por el modo mecánico y falto de comprensión al aprender los contenidos matemáticos.

Pérez-Tyteca y Castro (2011), la consideran como un estado afectivo caracterizado por la ausencia de confort, la cual se manifiesta mediante una serie de "síntomas", como son: tensión, nervios, preocupación, inquietud, irritabilidad, impaciencia, confusión, miedo y bloqueo mental.

Tomando en cuenta las definiciones anteriores, en este trabajo se concibe que la ansiedad matemática está constituida por los nervios, tensión, stress, bloqueo mental, síntomas físicos como sudor en las manos, dolor de estómago, dolor de cabeza, entre otros, que posee un estudiante al trabajar contenidos matemáticos.

\section{Estudios sobre ansiedad matemática.}

De acuerdo con Pérez-Tyteca (2012), una de las líneas de investigación sobre ansiedad matemática es la que estudia su relación con algunos factores como el género, rendimiento académico o escogencia de la carrera.

En relación con el género, algunos estudios (Hyde, Fennema, Ryan, Frost \& Hopp, 1990; Hembree, 1990; Pérez-Tyteca, 2012; Valero, 1999), manifiestan que las mujeres son más ansiosas que los hombres. Sin embargo, otras investigaciones (Reyes, 1984; Perina, 2002), discrepan con lo anterior y mencionan que las diferencias podrían deberse a que las mujeres son más sinceras al expresar sus sentimientos de ansiedad, mientras que los hombres son más reservados a externar dicha característica.

También se ha estudiado la relación entre la ansiedad matemática y la escogencia de la carrera universitaria. Al respecto, Tobias y Weissbrod (1980); Ashcraft (2002); y Furner y Berman (2003), mencionan que esta podría condicionar a los estudiantes a escoger una carrera universitaria que no incluyan cursos de matemática por el rechazo que sienten hacia esta disciplina. 
Por otra parte, Pérez-Tyteca, Castro, Segovia, Castro y Fernández (2007), añaden que un alto grado de ansiedad matemática dificulta el aprendizaje de ejercicios con mayor complejidad. En este sentido, los estudios de Bursal y Paznokas (2006) y Pérez-Tyteca (2012), compararon la ansiedad matemática y la autoconfianza, encontrando que entre mayor ansiedad matemática presente el estudiante, menor es su autoconfianza al resolver ejercicios de matemáticas.

Solazzo (2007) realizó un estudio sobre algunos factores ligados con el rendimiento, como son las habilidades de cálculo matemático y habilidades de razonamiento matemático. De acuerdo con el estudio, ambas están correlacionadas negativa y significativamente con la ansiedad matemática. Gliner (1987), también estudió factores como la cantidad de cursos de matemática que el alumno ha realizado, sexo, edad, ortografía, capacidad de expresión y ansiedad matemática, y su relación con el rendimiento académico. Este investigador encontró que el sexo, capacidad para expresarse, número de cursos que lleve en el semestre y ortografía, causan ansiedad matemática, pero no así el rendimiento académico.

Con respecto al rendimiento académico, se ha encontrado que la ansiedad matemática presenta una correlación negativa y significativa con esta variable, de manera que entre mayor nivel de ansiedad matemática, menor será el rendimiento académico (Aiken, 1970; Hembree, 1990; Reyes, 1984; y Pérez-Tyteca, 2012).

En esta misma línea, Eisele (1999) realizó un análisis de regresión múltiple con el fin de determinar la relación entre la ansiedad matemática, los estilos de aprendizaje y el rendimiento en una muestra de 150 estudiantes universitarios. Encontró que siete estilos de aprendizaje explican el 29.9\% del cambio en el nivel de ansiedad y que cinco estilos de aprendizaje explican el $29.6 \%$ del rendimiento.

Por otra parte, Rahim y Koeslag (2005) estudiaron la relación entre la edad y la ansiedad matemática. Encontraron que los estudiantes de mayor 
edad presentan menor ansiedad matemática en relación a los más jóvenes (entre 20 y 24 años de edad). Sobre este mismo tema, Malinsky, Ross, Pannels y McJunkin (2006) investigaron la relación de la ansiedad matemática con factores como género y edad. Los autores trabajaron con 481 estudiantes, con edades menores a 19 años y mayores a 30. Encontraron una relación entre la ansiedad matemática y la edad, pero no se pudo afirmar que entre mayor edad presente el estudiante, mayor será su ansiedad matemática.

Por último, Pérez-Tyteca (2012) sostiene que actualmente se trabaja la ansiedad matemática y la ansiedad ante los exámenes como dos conceptos claramente distinguidos, siendo esta la percepción por la cual aboga la autora. Además, Perina (2002) afirma que tanto la ansiedad matemática como la ansiedad ante los exámenes se encuentran relacionados, pues si un estudiante manifiesta ansiedad matemática, entonces es esperado que sufra ansiedad ante los exámenes.

\section{Estudios realizados en Costa Rica.}

Como se puede constatar, la Ansiedad Matemática es un campo de interés en Didáctica de la Matemática; sin embargo, ha sido poco abordada en Costa Rica. Al respecto se mencionan los estudios realizados por Meza, Agüero, Suárez y Schmidt (2014); Mena (2014); Castillo y Picado (2014) y Corrales (2014). A continuación se explican brevemente cada uno de estos estudios y algunos de sus resultados.

En el estudio de Meza et al., (2014), se analizó el nivel de ansiedad matemática de los estudiantes de la educación media costarricense. Para ello se aplicó el instrumento creado por Fennema y Sherman (1976) a una muestra no probabilística de 3725 estudiantes provenientes de 35 colegios públicos del país.

El estudio concluyó que aproximadamente el $60 \%$ de los estudiantes presentan niveles de ansiedad matemática bajos y que solamente un 3,4\%, muestran niveles de ansiedad matemática altos. Además, resultó que las mujeres presentan, aunque de forma moderada, mayor nivel de ansiedad 
matemática que los hombres y que los estudiantes de Educación Diversificada poseen un mayor nivel de ansiedad matemática que los estudiantes de Tercer Ciclo.

En la investigación realizada por Mena (2014), se estudió la ansiedad matemática de los estudiantes matriculados en los cursos de Matemática General, Cálculo Diferencial e Integral y Ecuaciones Diferenciales del Instituto Tecnológico de Cota Rica durante I Semestre 2013 y se aplicó el instrumento creado por Fennema y Sherman (1976). Mena concluye que el $85 \%$ de los estudiantes presentan un nivel de ansiedad matemática bajo o muy bajo y que las mujeres son más ansiosas que los hombres. Así mismo, determinó que existen diferencias significativas entre los niveles de ansiedad matemática de los estudiantes de Matemática General y los estudiantes de Cálculo Diferencial e integral y de Ecuaciones Diferenciales.

Corrales (2014) también estudió el nivel de ansiedad matemática presente en estudiantes de secundaria. Para ello aplicó el instrumento creado por Fennema y Sherman (1976) a estudiantes de tres colegios académicos nocturnos costarricenses. La investigación concluye que el $1.2 \%$ de los estudiantes presentó un nivel de ansiedad matemática muy alto, que las mujeres son más ansiosas que los hombres y que los estudiantes de segundo año de secundaria (14 años) presentan mayor nivel de ansiedad matemática que sus compañeros de primero, tercero y quinto año de secundaria.

Por último, en la investigación realizada por Castillo y Picado (2014) se estudió la ansiedad matemática en estudiantes de colegios técnicos de la educación media costarricense. Los resultados del estudio muestran que los estudiantes, en su mayoría, presentan un nivel de ansiedad matemática muy bajo (59\%) y que las mujeres poseen mayor nivel de ansiedad matemática que los hombres. Además, resultó que existen diferencias estadísticamente significativas entre el nivel de ansiedad matemática y el colegio de procedencia de los estudiantes. 


\section{El componente emocional en la Enseñanza de la Matemática.}

Algunos autores (McLeod, 1992; Guerrero, Blanco y Castro, 2001), confirman el incremento en los últimos años de los trabajos publicados sobre la parte afectiva del ser humano y su relación con el proceso de enseñanzaaprendizaje de las matemáticas, refiriéndose a la parte afectiva, a las actitudes, emociones y creencias del individuo. al respecto Silver (1985), en sus investigaciones sobre resolución de problemas, coloca las creencias de los estudiantes sobre la matemática como toda una disciplina.

Guerrero, Blanco y Castro (2001), muestran que las creencias, las actitudes y los pensamientos influyen directamente en las emociones y en los sentimientos. Además, agregan que de acuerdo a nuestra propia percepción y evaluación de los acontecimientos se llevan a cabo los cambios en nuestras emociones y en nuestro comportamiento.

Asimismo, el componente afectivo juega un papel sumamente importante en el aprendizaje exitoso de los estudiantes en el campo de las matemáticas, y en algunas ocasiones, estos aspectos afectivos están ligados al alumno y son difíciles de desplazar (Gil, Blanco y Guerrero, 2005).

Al igual que las creencias y las actitudes, la ansiedad matemática es un tema afectivo de gran peso en la educación matemática. Ahora bien, para hablar de ansiedad matemática es necesario abordar primeramente el concepto de ansiedad en general. A continuación se presentan algunas definiciones dadas al respecto.

\section{Niveles de Ansiedad Matemática}

De acuerdo con Pérez-Tyteca (2012), los niveles de AM se pueden clasificar de la siguiente manera:

- AM muy baja: Puntuación media menor a 1.5

- AM baja: Puntuación media entre 1.5 y 2.49

- AM media: Puntuación media entre 2.5 y 3.49

- AM alta: Puntuación media entre 3.5 y 4.49

- AM muy alta: Puntuación media mayor o igual a 4.5 
Para obtener el puntaje de cada estudiante, se toman en cuenta las preguntas del instrumento que miden la AM. Estas preguntas tienen escala tipo likert con opciones de respuesta de 1 a 5 ; los valores seleccionados por los estudiantes se suman y el resultado se divide entre el número total de preguntas. Estas fueron las categorías utilizadas en la presente investigación.

El objetivo de este trabajo consistió en determinar la relación entre ansiedad matemática y las variables género, rendimiento académico y tipo de colegio, en estudiantes del curso MAT-001 Matemática General de la Universidad Nacional de Costa Rica.

\section{Método}

\section{Tipo de investigación.}

Este estudio se considera de tipo descriptivo correlacional según Hernández, Fernández y Baptista (2010) ya que su propósito es evidenciar y describir las relaciones entre los puntajes obtenidos de las variables ansiedad matemática y género, rendimiento académico, cantidad de veces que ha llevado el curso y tipo de colegio, en estudiantes del curso MAT-001 Matemática General de la Universidad Nacional de Costa Rica. Además, el diseño fue descriptivo correlacional bivariado pues pretendía determinar las relaciones entre las variables antes mencionadas (Sánchez \& Reyes, 2002).

\section{Participantes.}

Los participantes del estudio son estudiantes matriculados en el curso MAT001 Matemática General, durante I Ciclo de 2015 en la Universidad Nacional de Costa Rica. La muestra total estuvo conformada por 472 estudiantes, de los cuales 203 eran hombres y 269 mujeres; 129 estudiantes estaban repitiendo el curso y 343 lo llevaban por primera vez. Los estudiantes pertenecían a las titulaciones de Ingeniería en Sistemas, Ingeniería Agrónoma, Biología, Economía, Teología, Enseñanza de la Matemática, Filosofía, Enseñanza de 
la Religión, Sociología, Planificación y Promoción Social, Administración de Empresas, Enseñanza de las Ciencias, Ingeniería en Ciencias Forestales, Ingeniería en Topografía y Catastro, Ingeniería en Gestión Ambiental, Comercio y Negocios Internacionales, Cartografía y Diseño Digital y Ciencias Geográficas.

A pesar de que se aplicó el instrumento a todos los grupos, el muestreo fue de tipo intencional ya que se tomaron en cuenta solamente los estudiantes que se encontraban en el aula al momento aplicar el instrumento.

\section{Instrumentos.}

El cuestionario utilizado corresponde a una adecuación de la escala elaborada por Hopko, Mahadevan, Bare y Hunt en el 2003, el cual fue modificado de acuerdo al contexto de Costa Rica y luego validado por expertos en Educación, Estadística, Psicopedagogía y Matemática Educativa. Además se aplicó una prueba piloto que permitió realizar algunas modificaciones al mismo.

El instrumento originalmente contaba con 9 preguntas que determinan el nivel de ansiedad matemática de los estudiantes; sin embargo, al tomar en cuenta las recomendaciones, se decidió incluir dos preguntas más para un total de 11. Cada uno de los ítems de los cuestionarios contó con cinco posibles opciones de respuesta enumeradas de la siguiente manera: 1, 2, 3, 4 y 5. La puntuación obtenida por cada estudiante en el cuestionario correspondió a la suma de los valores marcados en cada pregunta, divido entre el número total de preguntas. Para la fiabilidad del instrumento se utilizó el Alpha de Cronbach dando como resultado un valor de 0.88 , el cual permite garantizar la fiabilidad de la escala.

\section{Procedimiento.}

El instrumento fue aplicado a todos los grupos del curso MAT-001 Matemática General, I Ciclo 2015, en la semana 14 de clases, esto con el fin de determinar 
el nivel de ansiedad matemática presente en los estudiantes en ese momento. Es importante mencionar que al momento de aplicar el cuestionario ya habían realizado dos exámenes parciales y faltaba únicamente la aplicación del tercer y último examen parcial.

Para aplicar el instrumento se acudió a cada uno de los grupos y se solicitó a los estudiantes que completaran dicha encuesta. Previamente, se dieron las instrucciones con el fin de evitar errores al momento de completarla. Por último, les indicó la importancia de contestar todas las preguntas de la forma más sincera posible y les mencionó que el instrumento era anónimo y confidencial.

No existió un tiempo determinado para la conclusión de la aplicación del instrumento, sin embargo, el tiempo aproximado fue de 6 minutos para completar la encuesta. Dado que el curso tenía una duración de 16 semanas, en la semana 14 ya se encontraban trabajando en el último contenido a evaluar en el tercer examen parcial.

\section{Resultados}

El fin de este trabajo era determinar el nivel de ansiedad matemática de los estudiantes matriculados en el curso de Matemática General de la UNA y establecer si existen diferencias significativas en relación con el (a) sexo; (b) rendimiento académico; (c) tipo de colegio del cual es egresado el estudiante; (d) horario del curso; y (e) cantidad de veces que el estudiante a cursado la materia.

Para determinar si las diferencias encontradas eran estadísticamente significativas se aplicó el Modelo Lineal General Univariante. El nivel de significancia escogido para las pruebas de hipótesis fue .05. Para verificar el cumplimiento de los supuestos paramétricos se construyeron gráficos para evidenciar que los datos presentan gran tendencia a una distribución normal y se utilizó la prueba de Levene para comprobar que los datos cumplían homocedasticidad $\mathrm{u}$ homogeneidad de varianzas $p=0.424$. 


\section{Estudio de ansiedad matemática.}

El puntaje promedio de ansiedad matemática de los estudiantes encuestados fue de 2.89, valor que se obtuvo sumando los datos correspondientes al puntaje promedio de cada alumno y dividiendo este monto entre el total de entrevistados. El mismo (2.89), correspondió a un nivel de ansiedad matemática media según Pérez-Tyteca (2012).

Para obtener el puntaje promedio de ansiedad matemática de cada estudiante, se tomó en cuenta de la pregunta 11 a la 21 del instrumento. Los valores seleccionados por los estudiantes se sumaron y el resultado se dividió entre 11 (total de preguntas del instrumento). En la Tabla 1 se indican las frecuencias y porcentajes de la cantidad de estudiantes distribuidos de acuerdo a cada nivel de ansiedad matmática.

Tabla 1.

Frecuencia y porcentaje de estudiantes en cada nivel de ansiedad matemática.

\begin{tabular}{lcc}
\hline Nivel de ansiedad matemática & Frecuencia & Frecuencia \\
\hline Ansiedad matemática muy baja & 21 & 21 \\
Ansiedad matemática baja & 131 & 131 \\
Ansiedad matemática media & 206 & 206 \\
Ansiedad matemática alta & 107 & 107 \\
Ansiedad matemática muy alta & 7 & 7 \\
\hline
\end{tabular}

\section{Estudio de ansiedad matemática según sexo.}

Se realizó un análisis de los niveles de ansiedad matemática presentes en hombres y mujeres con el fin de observar si alguno de los géneros presentan mayores niveles de ansiedad al enfrentarse a tareas matemáticas. Al respecto se realizó un contraste de hipótesis y se encontró que las diferencias son estadísticamente significativas $p=.001$ con un tamaño del efecto (eta parcial al cuadrado) igual a .03. En la Tabla 2 se muestran los estadísticos descriptivos de la variable ansiedad matemática según el género. 
Tabla 2.

Estadísticos descriptivos de la ansiedad matemática según género.

\begin{tabular}{lccccc}
\hline Género & Media & $\mathrm{N}$ & Desviación típica & Mínimo & Máximo \\
\hline Hombre & 2.74 & 203 & 0.84 & 1.00 & 5.00 \\
Mujer & 3.00 & 269 & 0.78 & 1.36 & 4.82 \\
Total & 2.89 & 472 & 0.82 & 1.00 & 5.00 \\
\hline
\end{tabular}

De acuerdo con la tabla anterior se puede observar que los hombres presentaron menor ansiedad matemática que las mujeres, lo cual se evidencia en el puntaje medio de los hombres que resultó ser menor que el obtenido para el conjunto de la muestra; mientras que el de las mujeres fue mayor. En la Figura 1 se muestra más evidente este comportamiento.

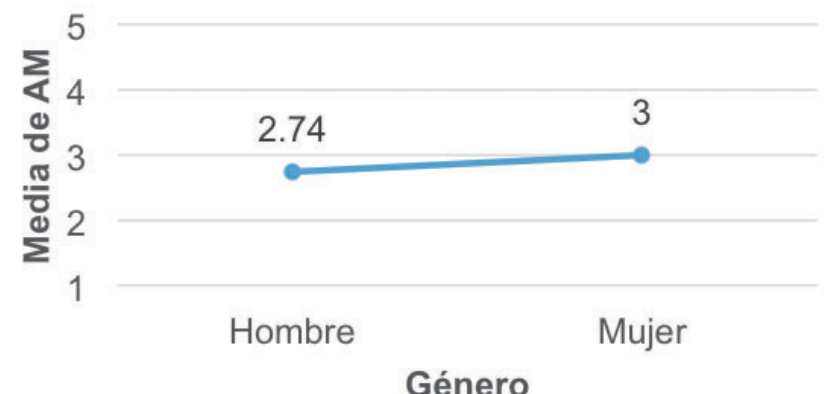

Figura 1. Ansiedad matemática según género.

\section{Estudio de ansiedad matemática según rendimiento académico}

Para analizar la variable rendimiento académico se realizó un contraste de hipótesis. Los resultados muestran que existe evidencia estadística suficiente para inferir que las diferencias encontradas entre el rendimiento académico y la ansiedad matemática son significativas $p<.001$, con un tamaño del efecto (eta parcial al cuadrado) igual a .09. 
En la Tabla 3 se muestran los estadísticos descriptivos de esta variable, los cuales reflejan que entre menor sea el rendimiento académico del estudiante mayor será su ansiedad matemática.

\section{Tabla 3.}

Estadísticos descriptivos de ansiedad matemática según rendimiento académico

\begin{tabular}{lccccc}
\hline $\begin{array}{l}\text { Nota obtenida en la } \\
\text { última }\end{array}$ & Media & $\mathrm{N}$ & Desviación típica & Mínimo & Máximo \\
\hline $\begin{array}{l}\text { Mayor que 0 pero } \\
\text { menor o igual a 25 }\end{array}$ & 3.56 & 22 & 0.67 & 1.91 & 4.73 \\
$\begin{array}{l}\text { Mayor que 25 pero } \\
\text { menor o igual a 50 }\end{array}$ & 3.06 & 91 & 0.86 & 1.18 & 5.00 \\
$\begin{array}{l}\text { Mayor que 50 pero } \\
\text { menor o igual a 70 }\end{array}$ & 3.00 & 164 & 0.69 & 1.45 & 4.64 \\
$\begin{array}{l}\text { Mayor que 70 pero } \\
\text { menor o igual a 85 }\end{array}$ & 2.68 & 124 & 0.79 & 1.00 & 4.82 \\
$\begin{array}{l}\text { Mayor que 85 pero } \\
\text { menor o igual a 100 }\end{array}$ & 2.54 & 71 & 0.92 & 1.00 & 4.73 \\
\begin{tabular}{l} 
Total \\
\hline
\end{tabular} & 2.89 & 472 & 0.82 & 1.00 & 5.00 \\
\hline
\end{tabular}

Como el rendimiento académico está compuesto por 5 valores y las diferencias son estadísticamente significativas, se aplicó los contrastes múltiples a posteriori mediante el método Scheffe para determinar entre cuáles niveles se daban estas diferencias.

Las diferencias encontradas entre el valor Mayor que 0 pero menor o igual a 25 y el valor Mayor que 70 pero menor o igual a $85 p<.001$, entre el valor Mayor que 0 pero menor o igual a 25 y el valor Mayor que 85 pero menor o igual a $100 p<.001$, entre el valor Mayor que 25 pero menor o igual a 50 y el valor Mayor que 70 pero menor o igual a $85 p=.008$, entre el valor Mayor que 25 pero menor o igual a 50 y el valor Mayor que 85 pero menor o igual a $100 p=.001$, entre el valor Mayor que 50 pero menor o igual a $70 \mathrm{y}$ el valor Mayor que 70 pero menor o igual a $85 p=.034$, entre el valor Mayor que 50 pero menor o igual a 70 y el valor Mayor que 85 pero menor o igual a 
$100 p=.004$, fueron estadísticamente significativas; las restantes diferencias no fueron significativas.

Esto indica que existen dos grupos significativamente homogéneos. El primero de ellos está compuesto por las calificaciones mayor o iguales que 0 y menores a 70; el segundo grupo por las calificaciones mayor o iguales a $70 \mathrm{y}$ menor o iguales a 100. En la Figura 2 se muestra mejor este comportamiento.

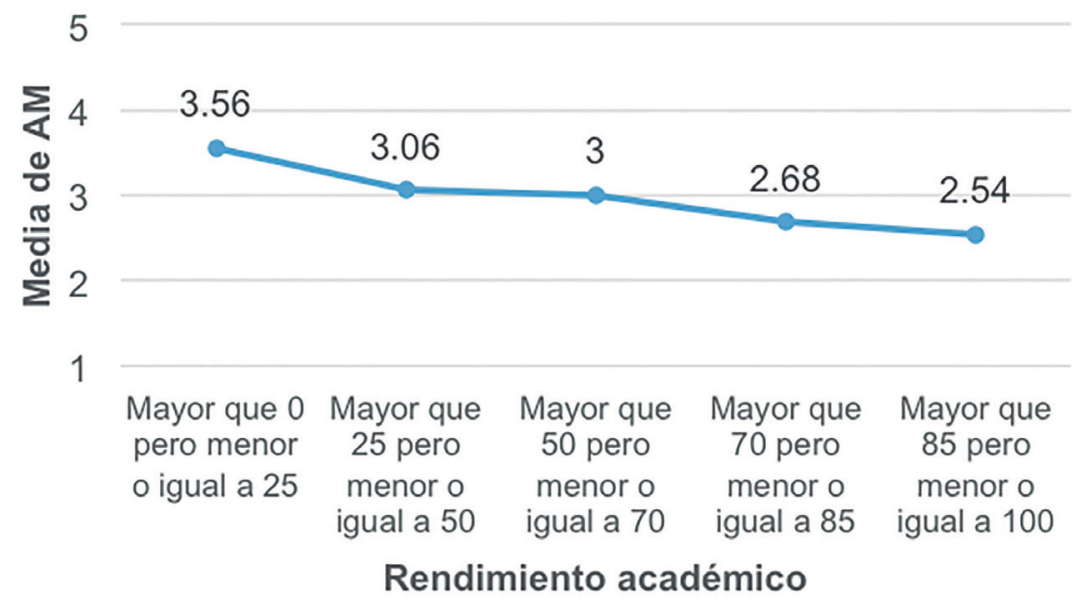

Figura 2. Ansiedad matemática según rendimiento académico.

\section{Estudio de ansiedad matemática según el tipo de colegio de procedencia.}

En cuanto al estudio del tipo de colegio de procedencia de los estudiantes, se realizó un contraste de hipótesis. Basándose en dicha prueba se observa que existe evidencia estadística suficiente para inferir que las diferencias encontradas entre el tipo de colegio y la ansiedad matemática son significativas $p<.001$, con un tamaño del efecto (eta parcial al cuadrado) igual a .04.

En la siguiente tabla se presentan los estadísticos descriptivos que muestran las diferencias encontradas. 


\section{Tabla 4.}

Estadísticos descriptivos de ansiedad matemática según el tipo de colegio.

\begin{tabular}{lccccc}
\hline Tipo de colegio & Media & $\mathrm{N}$ & Desviación Típica & Mínimo & Máximo \\
\hline Público & 2.97 & 358 & 0.80 & 1.00 & 5.00 \\
Privado & 2.60 & 106 & 0.80 & 1.09 & 4.45 \\
Otro (BxM) & 3.05 & 8 & 1.03 & 1.45 & 4.27 \\
Total & 2.89 & 472 & 0.82 & 1.00 & 5.00 \\
\hline
\end{tabular}

La Tabla 4 evidencia que los egresados de colegios privados presentaron menor ansiedad matemática que sus compañeros. Además, estos estudiantes obtuvieron un puntaje menor a la media de toda la muestra; mientas que las otras dos modalidades (público y bachillerato por madurez) muestran un puntaje mayor a la media de la muestra. Esta variable no fue encontrada en la literatura indagada. En la Figura 3 se específica el comportamiento encontrado.

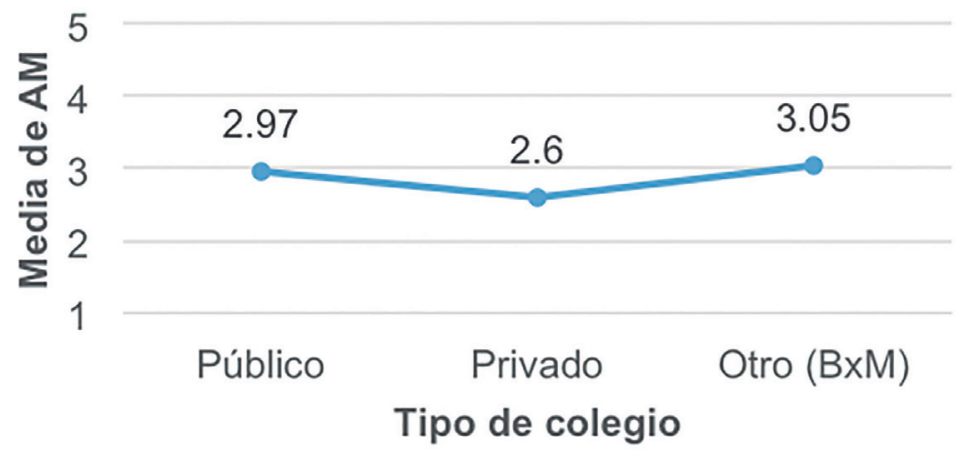

Figura 3. Ansiedad matemática según el tipo de colegio 


\section{Discusión}

De los resultados obtenidos en el análisis estadístico de los datos se puede concluir que los estudiantes de la muestra evidencian un nivel medio de ansiedad matemática. De acuerdo con Pérez-Tyteca (2012), lo ideal sería que los estudiantes que escogieron una titulación con mínimo una materia de matemáticas no presenten ansiedad hacia esta asignatura. Este resultado evidencia la existencia de una problemática y es un primer paso con el fin de trabajar posteriormente en estrategias de intervención que permitan manejar la ansiedad matemática adecuadamente en el salón de clase, tanto por parte del profesor, como por parte de los estudiantes.

También las pruebas de hipótesis realizadas permitieron establecer diferencias estadísticamente significativas entre la ansiedad matemática y las variables género, rendimiento académico y tipo de colegio de procedencia del estudiante.

Con respecto al género, se encontró que existen diferencias estadísticamente significativas en la ansiedad matemática presentes entre hombres y mujeres, siendo las mujeres las que muestran mayor ansiedad matemática; sin embargo, Perina (2002) y Reyes (1984) mencionan que estas diferencias pueden deberse a que las mujeres son más propensas a admitir que sufren de ansiedad, mientras que los hombres son más reservados al respecto. De igual forma, los resultados coinciden con las investigaciones realizadas por Eshaq (2006), Hembree (1990); Hyde, Fennema, Ryan, Frost y Hopp (1990); Pérez-Tyteca (2012) y Valero (1999). Este ha sido un primer acercamiento al comportamiento de la ansiedad matemática en hombres y mujeres del curso MAT-001 Matemática General de la Universidad Nacional en Costa Rica, por lo que se considera importante estudiar más a fondo este acontecimiento con el fin de determinar sus causas y la manera de disminuirlo.

En relación con el rendimiento académico, esta investigación concuerda con los resultados de Aiken (1970); Karasel, Ayda y Tezer (2010); Hembree (1990), Reyes (1984) y Pérez-Tyteca (2012) al afirmar que existe una 
relación negativa entre estas variables, ya que entre menor sea el rendimiento académico del estudiante, mayor ansiedad mostrará ante tareas matemáticas. También se encontró la formación de dos grupos significativamente homogéneos, el primero formado por las calificaciones mayores o iguales que 0 y menores a 70 y el segundo grupo constituido por las calificaciones mayores o iguales a 70 y menor o iguales a 100. Esta formación indica que las medias de ansiedad matemática de los estudiantes con calificaciones mayores o iguales a 70, son muy similares. Esta misma situación se presenta entre los estudiantes con calificaciones menores a 70, pues la formación de este grupo indica que las medias de ansiedad matemática en estos estudiantes son muy similares también. Se considera pertinente trabajar más a fondo este comportamiento. Curiosamente se divide entre estudiantes que aprueban y estudiantes que no. Sería oportuno indagar más sobre esas características similares presentadas por los estudiantes en cada uno de estos grupos.

En cuanto al tipo de colegio de procedencia, se encontró que los estudiantes egresados de colegios privados obtuvieron una ansiedad matemática significativamente menor que los provenientes de colegios públicos o de la modalidad de bachillerato por madurez. Se realizó una revisión de literatura relacionada con esta variable y no se encontraron estudios que la incluyeran, por lo que la investigación aporta un nuevo resultado a las investigaciones sobre ansiedad matemática. Este resultado es valioso en el sentido de que se deben realizar nuevos estudios que aborden las causas por las cuales los estudiantes de colegios públicos presentan mayor ansiedad matemática. Se recomienda incluso al Ministerio de Educación Pública de Costa Rica indagar en el trabajo que han realizado en el área de la matemática, pues son sus estudiantes quienes más sufren de ansiedad al desarrollar tareas matemáticas.

Por último, es necesario realizar otros estudios que aborden la ansiedad matemática y otras variables, con el fin de profundizar al respecto y verificar los resultados obtenidos en esta investigación. También se recomienda tomar en cuenta otros constructos del dominio afectivo como lo son la autoconfianza 
del estudiante o la utilidad que este le otorgue a la matemática; se considera importante profundizar en comparaciones entre la ansiedad matemática y estos constructos tomando en cuenta las variables mencionadas e incluso algunas otras.

\section{Referencias}

Aiken, L. (1970). Attitudes toward mathematics. Review of Educational Research, 551-596. doi: 10.3102/00346543040004551

Alegre, A. (2013). Ansiedad ante exámenes y estrategias de aprendizaje en estudiantes de secundaria de Lima Metropolitana. Propósitos y Representaciones, 1(1), 107-130. doi: 10.20511/pyr2013.v1n1.9

Ashcraft, M. H. (2002). Math anxiety: Personal, educational, and cognitive consequences. Current Directions in Psychological Science, 11(5), 181185. doi:10.1111/1467-8721.00196

Ashcraft, M. H., \& Krause, J.A. (2007). Working memory, math performance, and math anxiety. Psychonomic bulletin \& review, 14(2), 243-248. doi: 10.3758/bf03194059

Bursal, M. P., \& Paznokas, L. (2006). Mathematics anxiety and preservice elementary teachers confidence to teach mathematics and science. School Science and Mathematics, 106 (4),173-180. doi: 10.1111/j.19498594.2006.tb18073.x

Castillo, H., \& Picado, A. (2014). Estudio de la Ansiedad Matemática en estudiantes de colegios técnicos de la educación media costarricense (Tesis de Licenciatura). Instituto Tecnológico de Costa Rica. Costa Rica.

Cohen L., \& Manion, L. (2002). Métodos de investigación educativa ( $2^{\mathrm{a}}$ ed.). Madrid: La Muralla.

Corrales, J. (2014). Estudio del nivel de "ansiedad matemática" en estudiantes de tres colegios académicos nocturnos costarricenses (Tesis de Licenciatura): Instituto Tecnológico de Costa Rica. Costa Rica.

Eisele, R. (1999). The relationship of math anxiety and educational cognitive style to achievement in a mathematics content course for preservice elementary teachers (Tesis doctoral). Indiana: Indiana State University, Terre Haute. 
Eshaq, H. (2006). Student anxiety toward mathematics as a factor in the choice of the scientific or the literary study track in Saudi Arabian secondary education in Jazan (Tesis doctoral). Arkansas: University of Arkansas.

Fennema, E., \& Sherman, J. A. (1976). Fennema-Sherman mathematics attitudes scales: Instruments designed to measure attitudes toward the learning of mathematics by females and males. Journal for research in Mathematics Education, 7(5), 324-326. doi: 10.2307/748467

Furner, J. M., \& Berman, B. T. (2003). Review of research: math anxiety: overcoming a major obstacle to the improvement of student math performance. Childhood education, 79(3), 170-174. doi: 10.1080/00094056.2003.10522220

Gavira, N. (2008). Análisis de las causas del alto índice de reprobación en la asignatura de matemáticas en el bachillerato. Trabajo presentado en el Primer Congreso Internacional de Educación Media Superior y Superior, Ciudad de México, México.

Gil, N., Blanco, L., \& Guerrero, E. (2005). El dominio afectivo en el aprendizaje de las matemáticas. Una revisión de sus descriptores básicos. Unión: Revista Iberoamericana de Educación Matemática, 2, 15-32.

Gliner, G. (1987). The relationship between mathematics anxiety and achievement variables. School Science and Mathematics, 87(2), 81-87. doi: 10.1111/j.1949-8594.1987.tb11679.x

Guerrero, E., Blanco, L., \& Castro, F. (2001). Trastornos emocionales ante la educación matemática (pp. 229-237). Cáceres: Pirámide.

Hackett, G. (1985). Role of mathematics self-efficacy in the choice of mathrelated majors of college women and men: A path analysis. Journal of Counseling Psychology, 32(1), 47-56. doi: 10.1037/0022-0167.32.1.47

Hembree, R. (1990). The nature, effects, and relief of mathematics anxiety. Journal for Research in Mathematics Education, 33-46. doi: $10.2307 / 749455$

Hernández, R., Fernández, C., \& Baptista, M. (2010). Metodología de la investigación. México: McGraw-Hill. 
Hopko, D., Mahadevan, R., Bare, R., \& Hunt, M. (2003). The abbreviated math anxiety scale (AMAS). Construction, validity, and reliability. Assesment, 10(2), 178-182. doi: 10.1177/1073191103010002008

Hyde, J. S., Fennema, E., Ryan, M., Frost, L. A., \& Hopp, C. (1990). Gender comparisons of mathematics attitudes and affect. Psychology of women quarterly, 14(3), 299-324. doi: 10.1111/j.1471-6402.1990.tb00022.x

Karasel, N., Ayda, O., \& Tezer, M. (2010). The relationship between mathematics anxiety and mathematical problem solving skills among primary school students. Procedia Social and Behavioral Sciences, 2(2), 5804-5807. doi: 10.1016/j.sbspro.2010.03.946

Lamas,H.(2015). Sobre elrendimiento escolar.Propósitosy Representaciones, 3(1), 313-386. doi: 10.20511/pyr2015.v3n1.74

Lang, P. (1968). Fear reduction and fear behaviour: Problems in treating a construct. Research in Psychotherapy, 90-103. doi: 10.1037/10546-004

Malinsky, M., Ross, A., Pannells, T., \& McJunkin, M. (2006). Math anxiety in pre-service elementary school teachers. Education, 127(2), 274 -280.

Marshall, G. (2000). Explaining mathematics anxiety in college students. A research project. The Mathematics Educator, 5(1/2), 108-116.

Mato, M., \& Muñoz, J. (2010). Efectos generales de las variables Actitud y ansiedad sobre el rendimiento en matemáticas en alumnos de educación secundaria obligatoria. Implicaciones para la práctica educativa. Ciencias Psicológicas, 4(1), 27-40.

McLeod, D. B. (1992). Research on affect in mathematics education: A reconceptualization. Handbook of research on mathematics teaching and learning, 575-596.

Mena, J. (2014). Estudio de la ansiedad matemática en los cursos Matemática General, Cálculo Diferencial e Integral y Ecuaciones Diferenciales del Instituto Tecnológico de Costa Rica en el I Semestre 2013 (Tesis de Licenciatura). Instituto Tecnológico de Costa Rica. Costa Rica.

Meza, L., Agüero, E., Suarez, Z., \& Schmidt, S. (2014). ESAM: Estudio de la ansiedad matemática en la educación media. Instituto Tecnológico de Costa Rica. Costa Rica. 
NCTM (2003). Principios y estándares para la educación matemática. Reston: Estados Unidos.

Pérez-Tyteca, P. (2012). La ansiedad matemática como centro de un modelo causal predictivo de elección de carreras (Tesis Doctoral) Granada: Universidad de Granada, España.

Pérez-Tyteca, P., Castro, E., Rico, L., \& Castro, E. (2011). Ansiedad matemática, género y ramas de conocimiento en alumnos universitarios. Enseñanza de las ciencias: revista de investigación y experiencias didácticas, 29(2), 237-250.

Pérez-Tyteca, P., Castro, E., Segovia, I., Castro, E., \& Fernández, F. (2007). Ansiedad Matemática de los alumnos que ingresan en la Universidad de Granada. Investigación en Educación Matemática XI, 171-180.

Pérez-Tyteca, P., \& Castro, E. (2011). La ansiedad matemática y su red de influencias en la elección de carrera universitaria. Investigación en Educación, 471-480.

Perina, K. (2002). The sum of all fears. Psychology Today, 35(6), 19-19.

Perry, A. B. (2004). Decreasing math anxiety in college students. College Student Journal, 38(2), 321.

Quiles, M. N. (1993). Actitudes matemáticas y rendimiento escolar. Comunicación, Lenguaje y Educación, 5(18), 115-125. doi: 10.1080/02147033.1993.10821078

Rahim, M., y Koeslag, M. (2005). Math anxiety in Junior/Intermediate preservice teachers. In Alan Regerson. Reform, Revolution and Paradigm shifts in Mathematics Education, 194-198.

Reyes, L. (1984). Affective variables and mathematics education. The Elementary School Journal, 84(5), 558-581. doi: 10.1086/461384

Richardson, F., \& Suinn, R. (1972). The mathematics anxiety rating scale: Psychometric data. Journal of Counseling Psychology, 19(6), 551-554. doi: $10.1037 / \mathrm{h} 0033456$

Silver, E. A. (1985). Teaching and Leaming Mathematical Problem Solving: Multiple Research Perspectives. Mahwah, NJ: Erlbaum. 
Solazzo, L. (2007). The role of gender, cognition, anxiety, and competence beliefs in predicting mathematics achievement (Tesis doctoral). Fordham University, New York.

Spielberger, C. D. (1972). Conceptual and methodological issues in anxiety research. Anxiety: Current trends in theory and research, 2, 481-493. doi: 10.1016/B978-0-12-657402-9.50013-2

Tobias, S., \& Weissbrod, C. (1980). Anxiety and mathematics: An update. Harvard Educational Review, 50(1), 63-70. doi: 10.17763/ haer.50.1.xw483257j6035084

Valero, L. (1999). Evaluación de ansiedad ante exámenes: Datos de aplicación y fiabilidad de un cuestionario CAEX. Anales de Psicología, 15(2), 223231.

WAECE-AMEI. (2003). El Diccionario Pedagógico. Hermex Ibérica. Recuperado de http://waece.org/diccionario/index.php 